SCIENTIFIC REPORT

\title{
Unrecordable pulsatile ocular blood flow may signify severe stenosis of the ipsilateral internal carotid artery
}

\author{
Y Barkana, A Harris, L Hefez, M Zaritski, D Chen, I Avni
}

Br J Ophthalmol 2003;87:1478-1480

Aim: To examine the relation between stenosis of the internal carotid artery (ICA) and pulsatile ocular blood flow (POBF). Methods: In 57 eyes of 30 patients who were referred for Doppler ultrasound examination of the ICA we measured $\mathrm{POBF}$ and analysed the correlation with degree of ipsilateral ICA stenosis.

Results: There was a significant negative correlation between POBF and ipsilateral ICA stenosis (Pearson correlation coefficient, $r=-0.516, p<0.0001$ ). In 14 eyes POBF could not be measured by the OBF tonometer, and in 11 of these cases $(79 \%)$ severe stenosis $(>75 \%)$ of the ipsilateral ICA was present. When these eyes were excluded from analysis, there was no correlation between POBF and ICA stenosis $(r=-0.02, p=0.91)$. Among these 43 eyes in which POBF could be measured it ranged 667-2095 $\mu \mathrm{l} / \mathrm{min}$ with a mean of $970.72 \mu \mathrm{l} / \mathrm{min}$.

Conclusion: Low or unrecordable POBF may signify severe stenosis of the internal carotid artery. POBF is not a direct reflection of ipsilateral ICA blood flow.

$\mathrm{T}$ he OBF system (OBF Labs Ltd, Malmesbury, UK) is one of two systems that have been introduced for the measurement of pulsatile ocular blood flow (POBF). The instrument repeatedly measures the intraocular pressure (IOP) with a pneumatic applanation tonometer during the cardiac pulse cycle and, based on IOP variation with time, calculates the pulsatile ocular blood flow. ${ }^{1}$ It has gained popularity for use in ocular blood flow studies because of its ease of use and good reproducibility. ${ }^{2-4}$ Recent clinical publications have reported significant abnormalities in POBF in patients with normal tension glaucoma (NTG), ${ }^{5}$ age related macular degeneration, ${ }^{6}$ retinitis pigmentosa, ${ }^{7}$ and following regional ocular anaesthesia. ${ }^{8}$

Stenosis of the internal carotid artery (ICA) has been associated with various ophthalmic disorders such as ocular ischaemic syndrome, glaucoma, and vascular occlusions. ${ }^{9} 10$

To further characterise the role of POBF in health and disease, in this work we examined the relation of POBF to ICA stenosis. This relation has not been systematically characterised previously.

\section{METHODS}

We recruited consecutive patients who had carotid Doppler ultrasound examination in our institute. Only patients who could be tested in the sitting position were referred for POBF examination. Patients with arrhythmia or nystagmus were excluded. In addition, any significant ocular abnormality revealed on slit lamp examination such as corneal scar, glaucoma, or previous surgery excluded that eye from the study.
Ultrasound Doppler examination of the ICA included the peak systolic velocity (PSV), end diastolic velocity (EDV), and estimated percentage stenosis.

Ocular pulse amplitude (PA) and POBF were measured by the OBF machine. All measurements were made by the same investigator (YB) using the OBF tonometer mounted on a slit lamp with the patient seated after topical anaesthesia with Benoxinate $\mathrm{HCl} 0.4 \%$. Values reported by the machine were average of five best pulses during approximately 10 seconds of probe applanation to the eye. If the machine could not detect five measurable pulses during that period on three consecutive attempts, we marked the result as zero, denoting it as unrecordable.

Correlation between OBF values and ipsilateral ICA USDoppler parameters was evaluated using Pearson correlation coefficient. SPSS for Windows software, version 10.0, was used for the analysis.

\section{RESULTS}

Fifty seven eyes of 30 patients were included in the study. There were 21 males and nine females with mean age of 66 (SD 12) years.

Figure 1 is a scattergram demonstrating values of POBF and degree of ipsilateral ICA stenosis. There was a significant negative correlation between these two parameters (Pearson correlation coefficient, $r=-0.516, \mathrm{p}<0.0001)$.

$\mathrm{POBF}$ was unrecordable by the OBF tonometer in 14 eyes, and in 11 of these cases $(78.6 \%)$ ultrasound showed ipsilateral severe ICA stenosis (greater than $75 \%$ of the lumen). When these 14 eyes were excluded from analysis, there was no correlation between POBF and degree of ICA stenosis $(r=-0.02, \mathrm{p}=0.91)$.

Among the 43 eyes in which OBF was recorded, it ranged from $667-2095 \mu \mathrm{l} / \mathrm{min}$ with a mean of 970.72 . Severe stenosis of the ICA was observed in six of the 43 eyes (14\%).

In 17 of the examined ICA we observed stenosis greater than $75 \%$ of the lumen; in 11 ipsilateral eyes, POBF could not be recorded by the machine $(64.7 \%)$.

In seven patients, there was severe ICA stenosis on one side only (Table 1). In five of these patients, POBF was unrecordable in the eye ipsilateral to the stenosis, and was

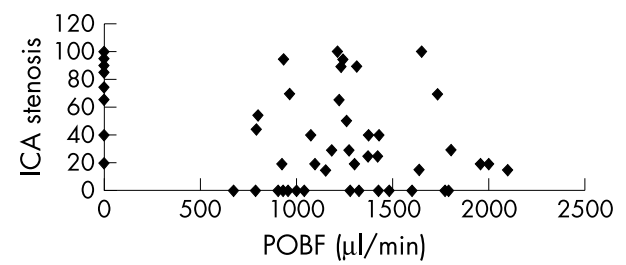

Figure 1 Scattergram showing percentage stenosis of the internal carotid artery (ICA) and ipsilateral pulsatile ocular blood flow (POBF) in 57 eyes of 30 patients included in this study. POBF of zero denotes inability to record POBF for that eye. 
Table 1 Stenosis of the internal carotid artery (ICA) and pulsatile ocular blood flow $(\mathrm{POBF})$ in seven patients with unilateral severe ICA stenosis

\begin{tabular}{|c|c|c|c|c|c|}
\hline \multirow[b]{2}{*}{ Patient } & \multirow[b]{2}{*}{ Age } & \multicolumn{2}{|c|}{ ICA stenosis (\%) } & \multicolumn{2}{|c|}{ POBF ( $\mu \mathrm{l} / \mathrm{min})$} \\
\hline & & Right & Left & RE & LE \\
\hline 1 & 80 & 90 & 40 & 0 & 1375 \\
\hline 2 & 62 & 20 & 85 & 921 & 0 \\
\hline 3 & 67 & 66 & 95 & 1226 & 0 \\
\hline 4 & 70 & 99 & 30 & 1649 & 0 \\
\hline 5 & 77 & 95 & 40 & 0 & 1428 \\
\hline 6 & 63 & 30 & 90 & 1809 & 1306 \\
\hline 7 & 73 & 100 & 30 & 0 & 1270 \\
\hline
\end{tabular}

normal on the contralateral side. In one patient, POBF was $1306 \mu \mathrm{l} / \mathrm{min}$ on the side with $90 \%$ ICA stenosis and $1809 \mu \mathrm{l} /$ min on the side with $30 \%$ ICA stenosis. In one patient POBF was $1650 \mu \mathrm{l} / \mathrm{min}$ on the side with $99 \%$ ICA stenosis and was unrecordable on the other side with only $30 \%$ stenosis.

In five patients, measurements were made before and after endarterectomy. In these cases POBF was not detectable preoperatively, and normalised following surgery (1185$1951 \mu \mathrm{l} / \mathrm{min}$, average 1410$)$.

Similar results were obtained when PA, PSV, or EDV were used for analysis, and consequently they are not included.

Heart rate, as measured by the OBF instrument, ranged from 42-90 beats per minute (mean 67.65 (13.9)).

\section{DISCUSSION}

There is current interest in determining whether reduced ocular blood flow is partly responsible for various eye diseases, such as glaucoma and age related macular degeneration. Proponents of the vascular pathogenetic hypothesis of open angle glaucoma (OAG) claim that chronic or intermittent decrease in blood flow to the optic nerve is partially responsible for the glaucomatous optic neuropathy. For example, the incidence of OAG has been reported to be higher in patients with lower perfusion pressure (blood pressure minus IOP). ${ }^{11}$ Progression of visual field loss has been related to greater than normal nocturnal decrease in systemic blood pressure. ${ }^{12}{ }^{13}$ Reduced blood flow has been observed by various methods in larger ocular arteries and nerve head microcirculation in patients with POAG and NTG. ${ }^{14-17}$

Accordingly, there is much interest in measuring ocular blood flow in humans non-invasively. The OBF tonometer reflects the main-pulsatile-portion of the choroidal blood flow, which in turn is the main contributor of ocular blood flow. ${ }^{18}{ }^{19}$ Thus, it may be used to study total ocular blood flow and blood flow to the optic nerve head.

We found a strong correlation between severe stenosis of the ICA and the inability of the OBF machine to record the pulsatile IOP. In certain situations, listed in the operating manual of the OBF tonometer, the machine may not record the pulsatile IOP of the eye. These include cardiac arrhythmia, poor patient fixation, dry eyes, and nystagmus. According to our results, significant stenosis of the ICA may be added to this list. After ruling out the above situations, in $64.7 \%$ of cases with significant ICA stenosis OBF could not be recorded. Presumably, the pulsatile component of blood flow to these eyes is severely reduced. Further research is needed to confirm this assumption and examine its implications for ocular disease.

Even more significant from a clinical standpoint, when OBF could not be recorded, there was a $79 \%$ chance of significant ICA stenosis on the ipsilateral side. This figure undoubtedly reflects the fact that our patients were recruited from the vascular ultrasound clinic and is higher than would be expected in the general population. Nevertheless, clinicians should consider performing cardiovascular examinations, specifically carotid ultrasound, in patients in whom pulsatile OBF can not be measured. Since the use of the OBF machine is fast and straightforward, it might even be incorporated into a screening programme of cardiovascular disease in high risk populations.

When we analysed only those eyes with recordable POBF, there was no correlation between POBF and ipsilateral ICA stenosis. Presumably, ocular blood flow is controlled by local autoregulation, and in cases of chronic severe stenosis may be maintained by collateral circulation. In this regard, it is interesting that Harris and co-workers reported that in glaucoma patients with stable visual fields, nocturnal ocular blood flow remains unchanged despite dips in systemic blood pressure. ${ }^{20}{ }^{21}$ In addition, invasive animal studies have shown that retinal and choroidal blood flow is maintained when systemic blood pressure or IOP are changed. ${ }^{22} 23$

The clinical relation between POBF and extra cranial blood vessels has not been thoroughly studied. There is one case report where an inter-eye difference in measured PA was attributed to a stenotic innominate artery, with equal values measured following the dilation of the stenotic vessel. ${ }^{24}$ Two cases were reported by Clarideg and James where POBF was measured before and after surgery for ICA stenosis. ${ }^{25}$ In one case POBF was unrecordable before surgery and $173 \mu \mathrm{l} / \mathrm{min}$ postoperatively. In the second case POBF increased significantly from 280 to $758 \mu \mathrm{l} / \mathrm{min}$. In our study we observed the normalisation of POBF in five cases following ICA surgery after being unrecordable preoperatively.

POBF values in our study were somewhat higher than previously reported in healthy eyes. Mori et al reported POBF values ranging from 290.7-1201.6 $\mu \mathrm{l} / \mathrm{min}$ with a mean of $593.3 \mu \mathrm{l} / \mathrm{min}$ in 80 normal subjects using the Langham OBF tonometer (Langham Ophthalmic Technologies, Timonium, MD, USA) ${ }^{26}$ Yang et al reported a range of 306-1645 with a mean of $775.9 \mu \mathrm{l} / \mathrm{min}$ in 83 healthy eyes using the OBF tonograph (OBF Laboratories, UK Ltd, Wilts, UK) which is similar to the one we used. ${ }^{3}$ POBF values in the present study ranged 667-2095 with a mean of $970.72 \mu \mathrm{l} / \mathrm{min}$.

In conclusion, we have demonstrated that inability to record POBF in healthy eyes may be related to severe stenosis of the ICA, and that severe ICA stenosis may signify a marked reduction in the pulsatile component of ocular blood flow. Clinicians using the OBF tonometer should be aware of this association and consider its implications for the general health of their patients. The significance of carotid artery stenosis to ocular blood flow in health and disease requires further investigation.

\section{Authors' affiliations}

Y Barkana, L Hefez, I Avni, Department of Ophthalmology, Assaf Harofe Medical Center, Beer Yaacov, Zerifin, Israel 
A Harris, Glaucoma Research and Diagnostic Center, Department of Ophthalmology, Indiana University School of Medicine, USA

M Zaritski, D Chen, Vascular Ultrasound Clinic, Assaf Harofe Medical Center, Beer Yaacov, Zerifin, Israel

Correspondence to: Yaniv Barkana, MD, Department of Ophthalmology, Assaf Harofe Medical Center, Beer Yaacov, Zerifin 70300, Israel; idityaniv@yahoo.com

Accepted for publication 7 April 2003

\section{REFERENCES}

1 Silver DM, Farrell RA. Validity of pulsatile ocular blood flow measurements. Surv Ophthalmol 1994;38(Suppl):S72-80.

2 Spraul CW, Lang GE, Ronzani $M$, et al. Reproducibility of measurements with a new slit lamp-mounted ocular blood flow tonograph. Graefes Arch Clin Exp Ophthalmol 1998;236:274-9.

3 Yang YC, Hulbert MF, Batterbury M, et al. Pulsatile ocular blood flow measurements in healthy eyes: reproducibility and reference values. J Glaucoma 1997:6:175-9.

4 Schmetterer L, Dallinger S, Findl O, et al. Noninvasive investigations of the normal ocular circulation in humans. Invest Ophthalmol Vis Sci 1998; 39:1210-20.

5 Fontana L, Poinoosawmy D, Bunce CV, et al. Pulsatile ocular blood flow investigation in asymmetric normal tension glaucoma and normal subjects. Br J Ophthalmol 1998;82:731-6

6 Mori F, Konno S, Hikichi T, et al. Pulsatile ocular blood flow study: decreases in exudative age related macular degeneration. $\mathrm{Br} J$ Ophthalmo 2001:85:531-3.

7 Schmidt KG, Pillunat LE, Kohler K, et al. Ocular pulse amplitude is reduced in patients with advanced retinitis pigmentosa. $\mathrm{Br} J$ Ophthalmol 2001;85:678-82.

8 Watkins R, Beigi B, Yates $M$, et al. Intraocular pressure and pulsatile ocular blood flow after retrobulbar and peribulbar anaesthesia. $\mathrm{Br} J$ Ophthalmol 2001;85:796-8.

9 Lyons Wait VA, Anderson SF, Townsend JC, et al. Ocular and systemic findings and their correlation with hemodynamically significant carotid artery stenosis: a retrospective study. Optom Vis Sci 2002;79:353-62.
10 Lawrence PF, Oderich GS. Ophthalmologic findings as predictors of carotid artery disease. Vasc Endovascular Surg 2002;36:415-24.

11 Leske MC, Wu SY, Nemesure B, et al. Incident open-angle glaucoma and blood pressure. Arch Ophthalmol 2002;120:954-9.

12 Graham SL, Drance SM. Nocturnal hypotension: role in glaucoma progression. Surv Ophthalmol 1999;43(Suppl 1):S10-6.

13 Graham SL, Drance SM, Wiisman K, et al. Ambulatory blood pressure monitoring in glaucoma. The nocturnal dip. Ophthalmology 1995;102:61-9.

14 Kerr J, Nelson P, O'Brien C. A comparison of ocular blood flow in untreated primary open-angle glaucoma and ocular hypertension. Am J Ophthalmol 1998;126:42-51.

15 Yamazaki Y, Drance SM. The relationship between progression of visual field defects and retrobulbar circulation in patients with glaucoma. Am J Ophthalmol 1997:124:287-95.

16 Nicolela MT, Hnik P, Drance SM. Scanning laser Doppler flowmeter study of retinal and optic disk blood flow in glaucomatous patients. Am J Ophthalmol 1996; 122:775-83.

17 Nicolela MT, Drance SM, Rankin SJ, et al. Color Doppler imaging in patients with asymmetric glaucoma and unilateral visual field loss. Am J Ophthalmol 1996;121:502-10

18 Langham ME, Farrell RA, O_Brien V, et al. Blood flow in the human eye. Acta Ophthalmol 1989;191(Suppl):9-13.

19 Krakau CE. A model for pulsatile and steady ocular blood flow. Graefes Arch Clin Exp Ophthalmol 1995;233:112-18.

20 Harris A, Evans D, Martin B, et al. Nocturnal blood pressure reduction: effect on retrobulbar hemodynamics in glaucoma. Graefes Arch Clin Exp Ophthalmol 2002;240:372-8.

21 Harris A, Spaeth G, Wilson R, et al. Nocturnal ophthalmic arterial hemodynamics in primary open-angle glaucoma. I Glaucoma 1997;6:170-4

22 Kiel JW, van Heuven WA. Ocular perfusion pressure and choroidal blood flow in the rabbit. Invest Ophthalmol Vis Sci 1995:36:579-85.

23 Sossi N, Anderson DR. Effect of elevated intraocular pressure on blood flow. Occurrence in cat optic nerve head studied with iodoantipyrine I 125. Arch Ophthalmol 1983;101:98-101.

24 Kaufmann C, Fierz A, Kollias SS, et al. Ocular pulse amplitude in a case of innominate steal syndrome. Am J Ophthalmol 2002;133:155-156.

25 Claridge KG, James CB. Ocular pulse measurements to assess pulsatile blood flow in carotid artery disease. $\mathrm{Br} J$ Ophthalmol 1994;78:321-3.

26 Mori F, Konno S, Hikichi T, et al. Factors affecting pulsatile ocular blood flow in normal subjects. Br J Ophthalmol 2001;85:529-30. 